la revue La revue pour l'histoire du CNRS

POUR LHISTOIRE DU CNRS $\quad 24 \mid 2009$

Soixante-dixième anniversaire du CNRS

\title{
Il était une fois, les sciences de la vie
}

comment assurer le progrès des connaissances?

Michel Morange

\section{(2) OpenEdition}

Journals

Édition électronique

URL : https://journals.openedition.org/histoire-cnrs/9058

DOI : $10.4000 /$ histoire-cnrs.9058

ISSN : 1955-2408

Éditeur

CNRS Éditions

Édition imprimée

Date de publication : 5 octobre 2009

ISSN : 1298-9800

Référence électronique

Michel Morange, « Il était une fois, les sciences de la vie », La revue pour l'histoire du CNRS [En ligne],

24 | 2009, mis en ligne le 05 octobre 2009, consulté le 20 mai 2021. URL : http://

journals.openedition.org/histoire-cnrs/9058; DOI : https://doi.org/10.4000/histoire-cnrs.9058

Ce document a été généré automatiquement le 20 mai 2021.

Comité pour l'histoire du CNRS 


\title{
Il était une fois, les sciences de la vie
}

\author{
Comment assurer le progrès des connaissances?
}

\section{Michel Morange}

1 La question de l'orientation de la recherche vers des objectifs ciblés est aujourd'hui directement posée. Quelle place laisser à la recherche fondamentale, « libre » ? Le débat s'enlise souvent dans des positions de principe. L'histoire montre que la recherche scientifique a toujours été orientée, plus ou moins directement, par la société dans laquelle elle était faite. La politique scientifique du CNRS n'a échappé, à aucun moment, à ce conditionnement. Mais l'histoire nous révèle aussi les conditions dans lesquelles cette orientation ne nuit pas au développement des connaissances fondamentales. Les sciences du vivant peuvent servir d'exemple. La biologie fonctionnelle a connu au moins trois phases de développement au cours du XXe siècle. De 1930 à 1960, des efforts considérables ont été faits pour décrire les mécanismes fondamentaux communs à l'ensemble des êtres vivants, soutenus par le développement d'un ensemble de technologies aujourd'hui familières à tous les biologistes : techniques d'électrophorèse, d'ultracentrifugation, etc.

De 1970 à 1990, grâce à la technologie du génie génétique issue de ces travaux, la description moléculaire du monde vivant progressa de manière spectaculaire, produisant de nouveaux résultats comme la découverte de la structure mosaïque des gènes, et de multiples applications biotechnologiques.

Depuis quelques années, les technologies de la génomique et de la post-génomique ont pris le relais : elles ont également pour ambition d'engendrer de nouveaux faits et de nouvelles applications, mais représentent aussi un retour vers des questions fondamentales sur l'organisation du vivant, sa dynamique, et la complexité des processus évolutifs.

4 De manière générale, dans les sciences du vivant comme dans toutes les sciences, le progrès des connaissances peut avoir quatre moteurs : la volonté de répondre à une question fondamentale, le développement de nouvelles technologies, l'apparition de faits nouveaux qui orientent la recherche dans une direction particulière, ou des objectifs précis, d'application médicale par exemple. 
5 Un seul de ces moteurs ne permet pas de faire avancer les sciences. De nouvelles technologies ne suffisent pas, de même que la volonté de guérir le cancer n'est pas, en soi, directement source de progrès. Mais il se produit assez naturellement une circulation entre ces quatre moteurs de la connaissance. De nouvelles technologies peuvent engendrer de nouvelles observations, sources d'applications ou d'interrogations fondamentales. De même, la recherche d'objectifs précis peut produire de nouveaux faits, à l'origine de nouveaux questionnements et du développement de nouvelles technologies, etc. C'est cette circulation, ce cercle vertueux, qui permet le renouvellement des systèmes expérimentaux et des modèles, moteur du progrès scientifique. Cette circulation reste bien visible lorsque l'on considère des domaines de recherche particuliers. Le cas des petits ARN régulateurs, siRNA (small interfering RiboNucleic Acid) et miRNA (micro RNA), est exemplaire. L'application de la technique de la transgenèse aux plantes mit en évidence le phénomène de la co-suppression. Les faits expérimentaux nouveaux ainsi produits conduisirent, par un chemin détourné, à la découverte des siRNA et des miRNA. Le rôle des ARN régulateurs est aujourd'hui une question centrale en biologie du développement, et des siRNA commencent à être utilisés en thérapeutique. Un objectif précis, appliqué, a produit des observations nouvelles, qui ont à leur tour engendré des travaux fondamentaux de recherche, parallèlement au développement d'applications médicales. De même, l'essor de la biologie synthétique, c'est-à-dire la modification rationnelle des êtres vivants pour leur faire acquérir de nouvelles fonctions et leur faire accomplir de nouvelles tâches, est aussi un moyen de tester les connaissances fondamentales acquises sur le vivant et, éventuellement, de les modifier et de les compléter.

6 L'histoire des sciences biologiques au $\mathrm{XX}^{\mathrm{e}}$ siècle montre ainsi que les moteurs $\mathrm{du}$ progrès scientifique ont été multiples et divers. Le déchiffrage du code génétique, un des résultats les plus fondamentaux de la biologie moléculaire, n'est-il pas venu d'un système expérimental mis au point pour comparer la synthèse des protéines dans les cellules normales et les cellules cancéreuses? Une recherche dirigée vers des objectifs précis peut contribuer à un progrès des connaissances fondamentales. À condition, cependant, que la circulation entre questions fondamentales, production de faits nouveaux, développement de nouvelles technologies et applications puisse s'opérer librement. Depuis sa création, le CNRS a permis cette circulation nécessaire au progrès des connaissances. Face aux réformes passées, actuelles et futures de la recherche, en particulier dans le domaine des sciences du vivant où les applications médicales, agronomiques et biotechnologiques des connaissances sont évidentes, il faut être vigilant à ce que cette circulation reste possible ; et que des obstacles institutionnels et des rigidités administratives ne viennent l'interrompre.

7 Analyse au microscope d'une boîte de cultures de cellules dans le cadre de la mise au point d'une nouvelle molécule (Dbait) dans le traitement des cancers multirésistants. () CNRS Photothèque/Sébastien Godefroy

8 Centrifugeuse avec rotor pour macroplaques. (c) CNRS Photothèque/Jérôme Chatin Modélisation tridimensionnelle illustrant le mode de reconnaissance entre un domaine protéique liant le zinc (sphère) et une double hélice d'ADN. (C) CNRS Photothèque/ Virginie Gervais 


\section{AUTEUR}

\section{MICHEL MORANGE}

Professeur à l'université Paris VI et à l'École normale supérieure, Michel Morange est biologiste moléculaire, historien des sciences et membre du Laboratoire de génétique moléculaire de l'ENS. 